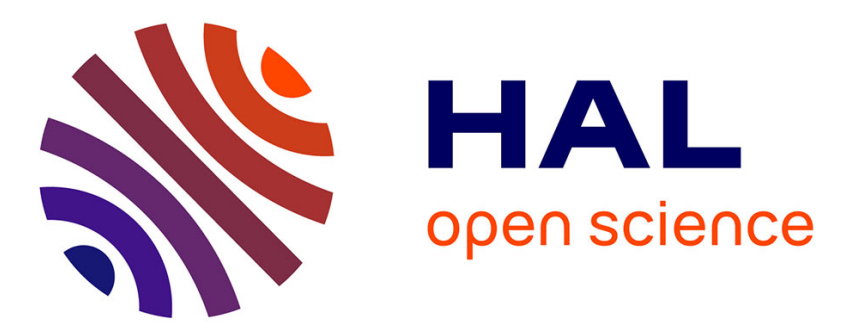

\title{
L'objection de conscience opposable, nouvelle stratégie légale et judiciaire des acteurs religieux aux Etats-Unis (2011-2018)
}

\author{
Blandine Chelini-Pont, Marie Gayte
}

\section{- To cite this version:}

Blandine Chelini-Pont, Marie Gayte. L'objection de conscience opposable, nouvelle stratégie légale et judiciaire des acteurs religieux aux Etats-Unis (2011-2018). Studies in Religion/Sciences Religieuses, 2019. hal-02364928

\author{
HAL Id: hal-02364928 \\ https://hal.science/hal-02364928
}

Submitted on 25 Nov 2019

HAL is a multi-disciplinary open access archive for the deposit and dissemination of scientific research documents, whether they are published or not. The documents may come from teaching and research institutions in France or abroad, or from public or private research centers.
L'archive ouverte pluridisciplinaire HAL, est destinée au dépôt et à la diffusion de documents scientifiques de niveau recherche, publiés ou non, émanant des établissements d'enseignement et de recherche français ou étrangers, des laboratoires publics ou privés. 
L'objection de conscience opposable, nouvelle stratégie légale et judiciaire des acteurs religieux aux Etats-Unis (20II-20 I8)
I-28

(C) The Author(s) / Le(s) auteur(s), 2019 Article reuse guidelines/ Directives de réutilisation des articles: sagepub.com/journals-permissions DOI: $10.1177 / 0008429819886729$ journals.sagepub.com/home/sr

(\$SAGE

\section{Marie Gayte}

Université de Toulon, Laboratoire Babel, France

\section{Blandine Chelini-Pont}

Université d'Aix-Marseille, LID2MS, France

Résumé : En mars 2010, Barack Obama fait adopter par le Congrès une réforme visant à élargir au maximum la couverture maladie des Américains. Parmi les points de la réforme, dans le cadre des plans collectifs, les employeurs ont l'obligation de fournir à leurs salariés un panier de soins préventifs dont le contenu, édicté par le ministère de la santé, inclut les moyens de contraception. Le ministère de la Santé a écarté la prise en charge des soins contraceptifs par les employeurs religieux au sens strict comme les Eglises, puis les organisations religieuses sans but lucratif dont les universités et les hôpitaux. Mais ces exemptions ne vont pas empêcher une levée de boucliers au sein de l'épiscopat catholique qui dénonce le contraceptive mandate comme une atteinte à la liberté religieuse et en appelle à la désobéissance civile. II est bientôt rejoint par de nombreuses organisations évangéliques, avec lesquelles il finit par faire cause commune pour défendre le droit à l'objection de conscience des chrétiens à accepter la contraception comme un soin pris en charge. Ce droit recouvre également leur refus de la légalité de l'avortement et du mariage homosexuel. Depuis 2010, plusieurs Etats, sur la base de cette posture et grâce à des majorités républicaines ont adopté des textes protégeant prioritairement la liberté religieuse incluant l'objection en cas de conflit de

\section{Corresponding author / Adresse de correspondance :}

Blandine Chelini-Pont, Aix-Marseille Universite, 5 Avenue Schuman, Aix-en-Provence, I3100, France.

Email: blandine.chelini-pont@univ-amu.fr 
conscience face à la loi. Cette présentation s'attachera à mettre en évidence les racines de l'objection de conscience dans le contexte religieux américain ainsi que sa direction actuelle. Elle dressera un premier état des lieux de l'efficacité, légale et judiciaire, de cette stratégie d'objection religieuse

Abstract: In March 2010, Congress adopted at Barack Obama's urging a healthcare reform aimed at offering health insurance to a majority of Americans. Among the points of the reform, for the collective plans, employers are required to provide their employees with a basket of preventive care. Its content, enacted by the Ministry of Health, includes contraceptive care. The Ministry of Health has dismissed contraceptive care by religious employers in the strict sense, such as churches, and eventually non-profit religious organizations, including universities and hospitals. But these exemptions will not prevent an outcry from the Catholic episcopate, which denounces the contraceptive mandate as an attack on religious freedom. Some bishops call for civil disobedience. They were soon joined by many evangelical organizations, making a common cause to defend the right for Christians to religious objection when the law is conflicting with their conscience. It concerns of course the contraceptive mandate but includes logically the legality of abortion and same sex marriage. Since 2010, several US states, thanks to Republican majorities, have adopted or tried to adopt bills protecting religious freedom as including religious objection in case of conscience conflicts with the law. This article will focus on highlighting the roots of conscious objection in the American religious context and its current direction. It will make an initial assessment of its legal and judicial results.

\section{Mots clés}

Acteurs religieux, Etats-Unis, objection de conscience, liberté religieuse

\section{Keywords}

Religious actors, United States, conscious objection, religious freedom

\section{Introduction}

Les droits des femmes et des homosexuels ont connu aux Etats-Unis depuis une quarantaine d'années des avancées sans précédents, ce qui a généré chez les religieux conservateurs le sentiment que leurs adversaires gagnaient du terrain de toute part. Ce sentiment s'est accentué depuis l'élection de Barack Obama en 2008, dont la présidence fut marquée par une accélération de ces mutations, avec notamment l'imposition d'un mandat contraceptif adopté dans le cadre de la réforme de santé de 2010 et la légalisation du mariage entre personnes de même sexe en 2015. La mobilisation des chrétiens engagés contre les évolutions sociétales qu'ils jugent contraires aux valeurs chrétiennes de l'Amérique, telles que le droit à l'avortement, la pornographie, l'interdiction de la prière à l'école, ne date pas d'hier, puisque ses premières manifestations remontent plus de quarante ans en arrière. Mais la réforme de santé a constitué un nouveau catalyseur qui a conduit les groupes et lobbies religieux à une autre approche dans leur mobilisation 
que nous pourrions qualifier de contestataire ou de résistante : après avoir tenté d'imposer une législation et une jurisprudence conformes à leurs convictions sur les questions morales et culturelles, en se fondant sur l'argument de leur caractère majoritaire dans la population, les chrétiens conservateurs se sont présentés à ce moment-là comme un élément minoritaire discriminé par la société agressivement laïque du début du millnaire, brandissant à ce titre l'objection de conscience comme leur bouclier et leur droit fondamental opposable à toute loi. Une partie non négligeable des Eglises américaines, dont la conférence nationale des évêques catholiques (USCCB) ont accrédité l'idée qu'en accordant des droits immoraux et contre nature à différents segments et contextes sociaux, c'est aux droits des chrétiens que l'on portait atteinte.

Cet article se propose donc, notamment par le recours à la théorie des mouvements sociaux, et plus particulièrement au concept de cadrage (framing), de tenter de mieux comprendre le tournant opéré par l'Eglise catholique - et d'autres acteurs religieux depuis une dizaine d'années dans la manière qu'elle a de conceptualiser ses luttes aux Etats-Unis.

\section{Quel cadrage pour mener la « guerre culturelle » ?}

Le cadrage peut se définir comme les « initiatives stratégiques conscientes prises par un groupe de personnes pour concevoir une compréhension partagée du monde et d'euxmêmes qui légitime et motive l'action collective $»{ }^{1}$. Les organisations, en présentant leurs exigences de manière à convaincre que leur cause est légitime, cherchent ainsi à obtenir des avantages des autorités et du public, la stratégie la plus efficace consistant à faire entrer en résonnance les préoccupations de leur mouvement avec des thèmes culturels et des valeurs communs à l'ensemble de la société ${ }^{2}$. Nous nous efforcerons donc tout d'abord de présenter brièvement l'histoire de la mobilisation des chrétiens conservateurs et surtout l'évolution de leurs stratégies de cadrage. Nous étudierons ensuite l'exemple précis de la conférence des évêques catholiques des Etats-Unis (USCCB) ; nous présenterons les circonstances ayant contribué à son adoption d'une stratégie de cadrage axée sur la défense de la liberté religieuse d'une minorité persécutée, et son corollaire, la revendication d'un droit à l'objection de conscience pour les chrétiens conservateurs. Au chapitre des réussites, le recours à ce cadrage particulier employé par d'autres groupes chrétiens, notamment les protestants évangéliques, a permis la constitution d'un œcuménisme de combat sans précédent entre des confessions dont on avait jusque-là annoncé maintes fois l'avènement, mais qui ne s'était jamais encore concrétisé. En outre, le discours de menaces sur la liberté religieuse a été repris par nombre de républicains et a débouché sur plusieurs réalisations concrètes, sur le plan législatif et sur le plan jurisprudentiel. Nous nous pencherons enfin sur la présidence Trump, jusqu'à présent une ère faste pour les tenants de ce cadrage.

Les conservateurs chrétiens, parfois désignés sous le vocable de « Nouvelle Droite chrétienne », forment un « mouvement social visant à mobiliser politiquement les chrétiens évangéliques et les autres chrétiens orthodoxes $»^{3}$. Cette mobilisation des droites religieuses est née à la fin des années 1970. Que ce soit par le biais d'agences religieuses telles Moral Majority ou, plus tard, la Christian Coalition, ou par celui d'Eglises directement, comme les Eglises catholiques ou mormones, elle vise 
historiquement à mobiliser politiquement les fidèles derrière des causes comme la lutte contre l'avortement, le mariage gay, l'euthanasie ... afin que les convictions des croyants sur les questions de morale et de culture servent de fondement à la législation s'appliquant à tous, croyants et non-croyants. Ce mouvement est né en réaction aux évolutions sociétales des années 1960 et du début des années 1970, dont l'interdiction de la prière dans les écoles publiques décidée par la Cour suprême en 1962, la légalisation par cette même Cour suprême de l'avortement en 1973, et plus généralement à la présence croissante de la pornographie dans les média. Ce mouvement s'est amplifié face aux bouleversements des rôles entre homme et femme au sein de la famille, dont un projet d'amendement visant à garantir l'égalité entre hommes et femmes en 1972 a incarné l'acmé. Tous ces éléments semblaient indiquer une nette sécularisation de la société américaine. Le combat des chrétiens conservateurs s'inscrit dans les « guerre culturelles » décrites par le sociologue James Davison Hunter dans son ouvrage de 1991, Culture Wars: The Struggle to Define America, comme une polarisation croissante de la société américaine autour de thèmes tels que le contrôle des armes à feu, l'avortement ou les droits des homosexuels.

Le mouvement des chrétiens conservateurs a connu plusieurs phases dans son histoire, cherchant d'abord à faire élire des présidents, puis se concentrant sur l'élection de politiques à des échelons inférieurs. Il a aussi connu des évolutions dans ses stratégies de cadrage, c'est-à-dire dans la manière qu'il a de présenter son combat, pour tenir compte des évolutions de l'opinion publique ${ }^{4}$. Dans les années 1970, quand la droite chrétienne émerge, le ton qui prédomine est plutôt triomphaliste. Le pays vient d'élire son premier président évangélique en 1976, et les chrétiens conservateurs se voient comme une « majorité morale », nom d'une des premières et plus célèbres organisations du mouvement, fondée en 1979. L'organisation Christian Voice pour sa part présente sa mission comme « faire d'une majorité chrétienne une démocratie chrétienne »" Les dirigeants du mouvement font valoir l'idée selon laquelle les conservateurs chrétiens sont une majorité puissante en lutte contre un tout petit groupe constitué d'élites progressistes ${ }^{6}$. A l'époque, évangéliques et catholiques blancs sont d'ailleurs majoritaires dans la population et l'élection de Ronald Reagan, dont ils se présentent comme des éléments clés, vient confirmer ce sentiment ${ }^{7}$. L'opinion publique les suit d'ailleurs sur un certain nombre de sujets, notamment la question des droits des homosexuels. A l'époque de la formation de la droite chrétienne, une majorité d'Américains juge l'homosexualité « immorale $»^{8}$ et il était donc possible de cadrer la lutte des droites chrétiennes contre les droits des homosexuels comme une lutte contre l'immoralité.

Si les évolutions sociétales ont rendu impératives la mobilisation des évêques comme mentionné supra, elles rendent également indispensables l'adoption d'un nouveau cadre. En effet, il devient impossible de présenter l'homosexualité comme immorale alors qu'une majorité d'Américains est en désaccord (le pourcentage jugeant l'homosexualité morale augmente ainsi rapidement, passant de $38 \%$ en 2002 à $56 \%$ en 2011$)^{9}$, tandis que les années 2010-11 marquent un tournant pour ce qui est du soutien au mariage gay, qui franchit la barre des $50 \%$ d'opinion favorable ${ }^{10}$. Il n'est donc plus possible de recourir à un cadrage reposant concomitamment sur l'immoralité et le caractère minoritaire du soutien aux comportements dénoncés (homosexualité, contraception), d'autant que l'on sait que la contraception jouit du soutien massif de la population américaine $(90 \%)^{11}, \mathrm{y}$ 
compris chez les catholiques, qui en plein milieu du débat sur la réforme de santé et le mandat contraceptif, estimaient à $42 \%$ que la contraception était morale, tandis que $43 \%$ jugeaient que cela n'avait rien à voir à la morale ${ }^{12}$.

Cette mise en minorité sur leurs positions clés se double d'un déclin démographique qui vient accentuer ce sentiment de marginalisation. La dernière grande enquête du Pew Research Center, réalisée en 2014, a ainsi révélé une diminution de la part des chrétiens dans la population entre 2007 et 2014, cette dernière passant de 78,4 à 70,6\%. Ce déclin touche dans une moindre mesure les évangéliques qui ont vu leur part baisser, de 26,3 à $25,4 \%$ et les catholiques de 23,9 à 20,8\%. Ce phénomène s'est accompagné d'une hausse remarquée des sans religion, groupe dont la part est passée en seulement de 16,1\% à $22,8 \%$ de la population ${ }^{13}$.

Les droites chrétiennes entreprennent alors un changement de cadrage de leur lutte, évolution déjà en germe dès les années 1990 et que les évêques catholiques vont reprendre à leur compte au moment de l'annonce du mandat contraceptif. Dès les années 1990, certaines organisations religieuses commencent à présenter leur action comme le combat d'une minorité religieuse persécutée par les institutions politiques et judiciaires, dans un pays désormais aux mains des " séculiers » avec des chrétiens blancs opprimés ${ }^{14}$. On commence alors à lire chez Pat Robertson, fondateur de la Christian Coalition, par exemple, des références au mouvement des Africains-Américains pour les droits civiques ainsi que des appels à la désobéissance civile ${ }^{15}$. Mais c'est Ralph Reed, le premier directeur général de la Christian Coalition, qui théorise le changement de cadrage dans un ouvrage de 1994, quand il explique qu'un groupe minoritaire peut plus facilement faire appel à la sympathie du public qu'un groupe majoritaire, qui peut facilement être perçu comme tyrannique ${ }^{16}$. De son côté, même Moral Majority avait commencé à la fin des années 1980 à tester ce cadrage sur la polémique de la prière dans les écoles publiques. Constatant la popularité déclinante de son combat dans l'opinion publique $^{17}$, elle abandonne l'angle moral et religieux pour adopter l'angle constitutionnel, présentant l'enjeu sous l'angle du respect des droits constitutionnels des élèves ${ }^{18}$.

Le recours à la rhétorique des droits des minorités religieuses opprimées par une majorité séculière s'est intensifié depuis une dizaine d'années ${ }^{19}$ et concerne la grande majorité des organisations religieuses conservatrices, que ce soit le Family Research Council, la conférence des évêques catholiques, ou la National Hispanic Christian Leadership Conference, qui toutes affirment que le gouvernement fédéral est coupable de violations de la liberté religieuse ${ }^{20}$. Le choix de cette stratégie de cadrage présente l'avantage supplémentaire, à une époque de sécularisation croissante de la population et de la société américaines, de permettre à la droite chrétienne d'élargir le public susceptible de soutenir son combat aux libertaires qui se méfient de toute intervention du gouvernement fédéral dans la vie des citoyens, ou même aux Américains soucieux du respect de la Constitution ${ }^{21}$.

Pour le célèbre pasteur Rick Warren, les défenseurs de la liberté religieuse sont à comparer aux acteurs du boycott des bus de Montgomery ${ }^{22}$, tandis que pour le directeur du Family Research Council, Tony Perkins, la greffière du Kentucky, Kim Davis, emprisonnée plusieurs jours pour avoir refusé de délivrer des licences de mariage à des couples homosexuels au nom de ses convictions religieuses, est comparable à Martin Luther King et Rosa Parks. ${ }^{23}$ Cette liberté religieuse « en danger » selon James Dobson, 
fondateur de Focus on the Family ${ }^{24}$, est mise à mal selon les dirigeants chrétiens en de nombreuses occasions. C'est le cas quand des photographes ou des pâtissiers chrétiens sont poursuivis en justice pour avoir refusé de photographier ou faire un gâteau pour le mariage de personnes de même sexe ; quand des médecins chrétiens redoutent de perdre la clause de conscience qui leur permet de ne pas pratiquer d'avortements ; quand des organismes catholiques spécialisés dans l'adoption ou le placement en famille d'accueil perdent leur financement fédéral car ils refusent de confier des enfants à des couples de même sexe. En février 2011, l'administration Obama publie une nouvelle régulation concernant le droit à l'objection de conscience religieuse des professionnels de santé. Cette mesure avait pour objectif de remplacer une norme datant de l'administration Bush fils. Avec la nouvelle norme, l'objection de conscience est limitée aux personnels de santé qui refuse de pratiquer des avortements ou des stérilisations, et ne peut plus être invoquée par les personnes au nom de leur croyances pour refuser de fournir la pilule, soigner des personnes homosexuelles atteintes du SIDA, ou effectuer des fécondations in vitro pour les lesbiennes ou les femmes seules ${ }^{25}$. Avec la réforme de santé, ce sont les écoles, hôpitaux ou universités chrétiens qui sont contraints de fournir une couverture santé comprenant la contraception à leurs salariés dans le cadre de la réforme de santé de 2010 .

\section{Le nouvel usage de la « liberté religieuse " par les évêques américains : historique d'une mobilisation}

Si une partie des catholiques engagés, parmi lesquels les puissants réseaux des religieuses américaines, Network et la Leadership Conference of Women Religious (LCWR), ont été très favorables à la réforme de santé voulue par Barack Obama, voire l'ont soutenue très activement, les évêques américains pour leur part y ont été farouchement opposés, et ce malgré leur volonté exprimée collégialement depuis 1919 de voir instaurer un système fédéral et universel de santé.

Ces derniers ont pratiqué un lobbying intense contre le projet de loi débattu au Congrès en 2009 pour que soit exclue toute possibilité de prise en charge de l'avortement dans le projet. Et de fait, l'avortement a été exclu par un amendement préventif durant les discussions. Finalement adoptée en mars 2010, cette loi avait pour objectif de réduire le nombre d'Américains dépourvus de couverture santé en les obligeant à en souscrire une, si besoin était grâce à des aides fiscales. La loi contraignait également les entreprises de plus de cent salariés à proposer une couverture santé à leurs salariés. Il incomba au ministère de la Santé de déterminer les prestations médicales devant faire l'objet d'une prise en charge, tâche que le HHS (Health and Human Services), acronyme du ministère de la santé, confia à l'Institut de Médecine, afin de dépolitiser la décision. Sur la base de ses recommandations, le ministère annonce en août 2011 que les contraceptifs présents dans la nomenclature de la Food and Drug Administration devaient être pris en charge, tout en fournissant une liste d'organisations religieuses (Églises, ordres religieux ... ) qui en seraient totalement exemptées. Pour les autres employeurs, ces décrets les contraignaient, sous peine de sanctions financières, à proposer une assurance santé obligatoire couvrant la prise en charge des pilules contraceptives, du stérilet et des pilules à effet abortif. Or, les personnels féminins travaillant dans des établissements tenus par les 
diocèses, les congrégations religieuses ou les œuvres caritatives et sociales, comme les universités, les hôpitaux, les maisons de retraite, les écoles, les centres sociaux, les foyers, n'étaient pas concernés par l'exemption de facto.

Devant le tollé que souleva le décret, le président Obama proposa, en février 2012, un aménagement supplémentaire : que ces frais-là soient pris en charge par les fournisseurs d'assurance et non les employeurs. Pour l'USCCB, le fait que des employeurs catholiques institutionnels dussent accepter dans l'assurance obligatoire de leurs employés d'éventuels contraceptifs et anti-gestatifs restait inadmissible. Ils continuèrent à accuser le gouvernement de violer la liberté religieuse des citoyens américains, protégée par le premier amendement de la Constitution.

Pour mobiliser les catholiques, diverses initiatives furent lancées par l'USCCB, toutes utilisant le cadrage de la violation de la liberté religieuse d'une minorité et l'invocation des droits constitutionnels bafoués. En septembre 2011, elle mit en place une «commission spéciale sur la Liberté religieuse », avec pour mission de « contrer l'offensive visant à neutraliser la religion dans la sphère publique $»^{26}$. En avril 2012, la commission fit paraître une déclaration intitulée « Notre première et plus précieuse liberté : déclaration sur la liberté religieuse », dans laquelle étaient recensées les violations et annoncée la tenue d'une « Quinzaine pour la liberté », dont le point d'orgue devait être le 4 juillet $2012^{27}$. Le président de la commission, Mgr William Lori, également archevêque de Baltimore, siège historique de l'Eglise catholique américaine, appela les catholiques à désobéir aux lois iniques. Devant la Chambre des représentants, il affirmera que l'égalité des Américains LGBT et l'accès à la santé reproductive étaient de « graves menaces pour la liberté religieuse ». Ces questions étaient « les dernières manifestations d'une tendance plus générale d'érosion de la liberté religieuse aux États-Unis ${ }^{28}$.

Plus généralement, les évêques américains dénoncent tout soutien politique à l'avortement, la contraception et désormais le mariage homosexuel, ce qui a été particulièrement manifeste lors de la campagne présidentielle de 2012. Les évêques, sans aller jusqu'à dire nommément de voter pour le candidat républicain, appellent les catholiques à voter pour le candidat qui défend la vie et la famille. Ils sont plus discrets sur les questions de justice sociale, traditionnellement associées au Parti démocrate. Certains prélats, quand ils prennent la parole à titre individuel, ne font pas de secret de leur préférence au moment de déposer leur bulletin dans l'urne. Ainsi, Mgr Paprocki, par ailleurs connu pour être l'un des trois évêques chargés en 2013 par la Congrégation pour la doctrine de la Foi d'assurer la tutelle de la principale fédération des religieuses américaines, la $\mathrm{LCWR}^{29}$, fait paraître un article à quelques jours du scrutin présidentiel. Il y critique le programme du Parti démocrate pour son soutien sans équivoque au droit à l'avortement ; il rappelle que « voter pour un candidat qui promeut des actions ou des comportements intrinsèquement mauvais et constituant un péché grave rend toute personne moralement complice et place le salut éternel de son âme en grave danger $»^{30}$. Quant au programme du Parti républicain, il ne comporte rien, selon lui, « qui ne soutienne ou encourage un mal intrinsèque ou un péché grave ${ }^{31}$. En avril 2012, l'évêque de Peoria, Daniel Jenky, compare le président Obama à Staline ou Hitler pour avoir violé la liberté religieuse ${ }^{32}$. Quant au cardinal de Chicago, Francis George, il invite le clergé à instruire les fidèles à la veille du vote sur leur responsabilité spirituelle. Enfin, Timothy Dolan, cardinal-archevêque de New-York, a été invité par le Parti républicain à 
prononcer la prière de clôture de sa convention nationale où Mitt Romney est officiellement investi candidat aux présidentielles. Dans un souci d'équité, Mgr Dolan participe également à la convention du Parti démocrate. La bénédiction qu'il prononça lors de la Convention démocrate fut l'occasion de dénoncer le mariage gay et l'avortement et de rappeler son attachement à « notre liberté la plus précieuse, la première », c'est-à-dire cette liberté religieuse mise à mal par l'Obamacare ${ }^{33}$. Mgr Dolan a par ailleurs porté plainte - comme d'autres évêques - contre l'Affordable Care Act au nom de l'archidiocèse de New York.

En parallèle de ces actions, sur le plan judiciaire, la Conférence dénonce les décrets d'application ; la congrégation des Petites Sœurs des Pauvres du Colorado, bientôt suivie de centaines d'institutions religieuses catholiques ; objectent auprès des Cours fédérales, à remplir le formulaire 700 du HHS les concernant, car cela les aurait rendues « complices du péché » de contraception. En décembre 2013, la juge de la Cour suprême, Sonia Sotomayor, leur garantit par une injonction temporaire, ce droit de déclarer au ministère de la Santé leur objection, le temps que la jurisprudence fédérale aille au bout de sa propre procédure ${ }^{34}$. Ce sont les ministres de la Santé qui sont alors les cibles de ces multitudes de plaintes : Kathleen Sebelius de 2009 à 2014, puis Silvia Mathews Burwell à partir de cette date.

L’argument de la liberté religieuse bafouée, utilisé pour cette " fronde publique », s'est révélé particulièrement efficace. Il a permis d'utiliser la notion de contrainte de conscience insupportable, pour une loi obligeant des employeurs religieux à lui être soumis en tant que loi commune, alors que cette loi permettait et protégeait des actions blocage chimique de l'ovulation humaine, arrêt chimique de la fécondation humaine, stérilité mécanique - allant à l'encontre de leur éthique convictionnelle, en tant que membres d'une Eglise spécifique.

Associer la contestation d'une loi civile au respect supérieur et injonctif de sa foi dans ses conséquences éthiques n'est pas une nouveauté dans l'histoire américaine, mais elle atteint avec le mandat contraceptif une ampleur sans équivalence. L'objection de conscience a concerné auparavant le refus par des chrétiens pacifistes de la conscription militaire ou du « devoir de tuer » sur les champs de bataille pour le cas des Quakers. Elle a aussi été utilisée dans le refus de prêter le moindre serment d'allégeance à la Nation, considéré comme de l'idolâtrie par les Témoins de Jéhovah. Une grande jurisprudence du XIXème siècle a par ailleurs fixé les limites de la liberté religieuse, quand le chef de l'Eglise mormone contesta l'interdiction du mariage polygame imposé à sa communauté par l'Etat fédéral, sous peine de dissolution. A cette occasion, la Cour suprême américaine a défini la liberté religieuse invoquée en défense par l'Eglise mormone, en fixant avec l'arrêt Reynolds v. United States (1878) les limites de son périmètre extérieur. La liberté de foi est absolue et incontrôlable en tant que croyance du for interne, mais limitable dans ses manifestations concrètes - en l'occurrence la polygamie - quand celles-ci ont comme conséquence de troubler gravement l'ordre civilisé (sic), car aucune exception « ne [pouvait] permettre qu'une doctrine religieuse soit supérieure à la loi du pays, et par conséquent à chaque citoyen de devenir une loi à lui tout seul $»^{35}$.

La grande jurisprudence Reynolds a posé les limites des comportements religieux libres dans le cadre fixé par la loi commune, pour autant elle n'a pas considéré son pendant inverse, c'est-à-dire les limites de la loi commune à « forcer » des croyants à agir 
contrairement à leur foi ou éthique. Or c'est bien cette perspective qui est utilisée depuis 2011 par les groupes religieux, en particulier les institutions catholiques, le tout médiatisé par l'USCCB, avec d'autant plus de force que ces défenseurs de l'exemption religieuse à la loi commune considèrent freiner l'intrusion de l'Etat dans les consciences. Ce faisant, la polémique, très violente dans son intensité, rentre dans un contexte plus général et participe de la guerre culturelle qui sévit dans le pays depuis les années soixante-dix.

Les fondements de l'affrontement culturel et politique américain tournent en effet autour de deux axes, que l'on pourrait appeler libéral d'un côté et chrétien de l'autre, avec des exigences en matière d'esprit public et de lois communes souvent aux antipodes. Dans cette polarisation, la sexualité et la reproduction humaine déplacées par l'évolution des mentalités et l'aval du droit dans le champ des libertés individuelles intimes, des droits des femmes et des homosexuels, sont devenues un enjeu majeur du débat public américain ${ }^{36}$. Les évêques catholiques, à l'encontre d'une longue tradition de réserve publique qui les a caractérisés jusqu'aux années 1960, sont devenus partie prenante du débat public par la lutte qu'ils ont entamée contre l'avortement légalisé, en exigeant également un positionnement clair sur ce sujet des élites politiques d'obédience catholique $^{37}$. Leur déclaration commune de 2003, Faithful Citizenship: A Catholic Call to Political Responsibility, insiste sur le fait que l'avortement n'est jamais moralement acceptable $^{38}$. En 2004, leur virulence à l'égard du candidat présidentiel démocrate John Kerry, catholique et pro-choice, a marqué les mémoires. Dans une autre déclaration de 2007 Forming Consciences for Faithful Citizenship, la Conférence des évêques identifie comme priorités le racisme, l'avortement, l'injustice sociale globale, mais insiste encore : prendre une vie innocente n'est pas une question parmi d'autres. Avec le racisme, l'avortement tient de la catégorie des maux intrinsèques ${ }^{39}$. Cette orientation, quoique très débattue dans la mise à jour de la déclaration en 2015 - nombre d'évêques souhaitaient, à l'instar de la position pontificale ${ }^{40}$, que les questions de l'immigration et de l'environnement, la politique des réfugiés, le contrôle des armes, le désarmement, la pauvreté mondiale soient positionnées comme prioritaires - est encore maintenue, associée au rejet du mariage civil homosexuel ${ }^{41}$.

En même temps que cette constance prise de position publique, les évêques ont également été favorables, depuis ces années 1970, à l'usage des tribunaux fédéraux comme à celui des chambres législatives, notamment la fédérale, pour protéger l'éthos de l'Amérique, un éthos chrétien sapé par des lois non chrétiennes face à un éthos libéral, attentif à protéger les libertés des individus, y compris dans leur comportement reproductif, affectif et sexuel. Ainsi, ils ont soutenu - avec d'autres - le passage d'un amendement fédéral sur le respect de la vie, puis d'un amendement fédéral sur la souveraineté de Dieu, puis d'un amendement fédéral sur le mariage, ${ }^{42}$ finalement transformé en loi, la loi de défense du mariage (DOMA). Cette dernière, votée en 1996, se retrouve entachée d'inconstitutionnalité par une décision de la Cour suprême en 2013. C'est à partir de ce champ de bataille légal que se développe le thème large de la défense de la liberté religieuse, comme arme de reconquête ou de reconstruction morale.

S'il fallait remonter à la source contestataire de la liberté de religion qui a conduit à la dernière étape contemporaine d'un droit à l'objection convictionnelle systématique, nous pouvons la trouver dans la jurisprudence Employment Division v. Smith de 
$1990^{43}$. Deux employés amérindiens, licenciés par une clinique pour avoir consommé du peyotl, certes en usage depuis des siècles dans les rituels de leur religion mais consommé sur leur lieu de travail, se sont vus refuser les allocations chômage par l'administration compétente de leur État, l'Oregon. La Cour suprême fédérale, sans même faire intervenir le principe dégagé dans la célèbre jurisprudence Yoder $^{44}$, considéra le refus comme justifié, les plaignants ayant été renvoyés pour consommation d'un produit hallucinogène interdit par la loi fédérale sur les narcotiques. À partir de cette affaire, une véritable coalition religieuse se met en place pour obliger la Cour - et l'ensemble du système législatif - à pratiquer un contrôle strict des lois et des situations qui pourraient entrer en conflit avec les convictions religieuses d'un individu.

Une révolution législative s'est dessinée qui a transformé les controverses autour de la défense de la liberté religieuse en mouvement politique «pro-religion » : la loi de rétablissement de la liberté religieuse (Religious Freedom Restoration Act ou RFRA), loi fédérale sur le rétablissement de la liberté religieuse votée en 1993, rend obligatoire le contrôle strict de l'intérêt impératif de l'État et l'usage de la solution la moins restrictive pour l'expression religieuse. La loi sur la protection de la liberté religieuse et des dons caritatifs (Religious Liberty and Charitable Donation Protection Act) de 1998, exclut les dons aux organismes religieux et caritatifs des recouvrements de créances et les intègre dans les dépenses autorisées par les mandataires en cas de faillite. Le Religious Land Use and Institutionalized Persons Act, également de 1998, garantit des protections accrues en faveur des groupes religieux et des personnes en matière foncière (privilèges fiscaux et de crédit, priorités d'achat et de vente) et en matière de respect des croyances religieuses dans le cadre des prisons et des hôpitaux psychiatriques.

La loi fédérale dite RFRA est adoptée à l'unanimité à la Chambre et avec trois votes contre au Sénat, comme une réponse directe à l'affaire Employment Division vs Smith de 1990. Elle a provoqué un changement majeur dans l'approche de la Cour suprême des entraves à la liberté de religion. Le cadre juridique qui en a résulté offre une protection finalement bien plus forte à la liberté de religion que ne le prévoit la Constitution et cela, au détriment des autres libertés. Désormais, si la Cour conclut qu'une loi impose un « fardeau substantiel » (substantial burden) à la liberté de religion d'un croyant, cette personne peut invoquer l'entrave - la contrainte à sa liberté religieuse - pour ne pas y être soumis, à moins que le gouvernement ou le service public fédéral ne démontre que la loi représente le moyen le moins restrictif (the least restrictive means) pour la poursuite d'un intérêt impératif. Ce nouveau standard, connu sous l'expression de strict scrutiny est extrêmement difficile à atteindre et rend a priori n'importe quelle loi fédérale vulnérable. Même si en 1997 la loi RFRA a été jugée inconstitutionnelle en cas d'application aux États, ${ }^{45}$ elle reste une arme puissante contre toute loi fédérale en général, dès lors que cette dernière toucherait d'une manière ou d'une autre la liberté religieuse. C'est exactement ce qui s'est passé avec l'Obamacare, surnom de l'Affordable Care Act.

\section{Du droit de refus de complicité au droit à la discrimination religieuse active : le résultat des mobilisations depuis $20 \mathrm{I}$ I}

Une des fruits du recours au cadrage de défense de la liberté religieuse d'une minorité chrétienne menacée par une société sécularisée est tout d'abord la formation d'une 
coalition œcuménique de combat regroupant les chrétiens conservateurs, maintes fois annoncée et sans cesse démentie dans les faits ${ }^{46}$ La parution du manifeste œcuménique conservateur Evangelicals and Catholics Together, co-rédigé par Richard John Neuhaus et Charles Colson en 1994, au-delà du symbole, n'avait pas suscité l'engouement escompté. En 2009 en revanche, la Déclaration de Manhattan, de Charles Colson, de l'universitaire catholique Robert George et du doyen de la faculté de théologie des baptistes du Sud, Timothy George, semble avoir eu un impact fort. Signée à l'origine par 150 dirigeants évangéliques de tout premier plan (tels que James Dobson, Tony Perkins et Leith Anderson), par des catholiques, dont 50 évêques, archevêques et cardinaux, y compris le président de l'USCCB, le document, qui appelle à lutter ensemble sur des sujets chers aux yeux de tous les signataires, comme l'avortement, le mariage gay et les violations de la liberté religieuse, appelle les fidèles à la « résistance aux attaques du gouvernement et de la culture contre la liberté religieuse ». Il les enjoint à la « désobéissance civile » contre les « lois iniques », comme celles « obligeant les croyants à reconnaître les unions entre deux personnes de même sexe comme un mariage », ou les « forçant à participer à des avortements ou des euthanasies » ${ }^{47}$. Depuis, les déclarations œcuméniques pour dénoncer les attaques à la liberté religieuse se sont multipliées. A titre d'exemple, en juillet 2012, cent signataires, dont Mgr Lori et Leith Anderson, président de la National Association of Evangelicals, Samuel Rodriguez de la National Hispanic Christian Leadership Conference, Matthew Harrison, président du Lutheran ChurchMissouri Synod, Alan Sears, président de l'Alliance Defending Freedom, Tony Perkins, président du Family Research Council et Richard Land, président du Southern Evangelical Seminary demandèrent au président Obama d'étendre les exemptions au mandat contraceptif aux individus et aux organisations qui le souhaitaient ${ }^{48}$. En mai 2015, quelques semaines avant que soit rendu l'arrêt de la Cour suprême légalisant le mariage homosexuel, les responsables chrétiens reprenaient leur plume pour parapher « We Stand in Solidarity to Defend Marriage and the Family and Society Founded Upon Them », dans lequel ils faisaient le serment de s'opposer à la « redéfinition du mariage ${ }^{49}$.

Cet œcuménisme conservateur semble jouir de l'imprimatur du Saint-Siège. En janvier 2012, peu après l'annonce du mandat contraceptif par le ministère de la Santé américain, Benoît XVI évoquait son inquiétude au sujet de «certaines tentatives [...] visant à limiter la liberté la plus précieuse aux Etats-Unis, la liberté de religion » et « d'efforts concertés visant à refuser le droit à l'objection de conscience aux institutions et laïcs catholiques concernant la coopération à des principes intrinsèquement maléfiques $»^{50}$. Le Saint-Siège invita d'ailleurs à une conférence sur la complémentarité entre homme et femme dans le mariage de nombreux dirigeants évangéliques, comme Tony Perkins, ou Alan Spears d'Alliance Defending Freedom (ADF) ${ }^{51}$.

L'ADF, agence et cabinet d'avocats fondée entre autre par l'évangéliques James Dobson, définit sa mission comme la défense de la liberté religieuse, du caractère sacré de la vie, du mariage et de la famille ${ }^{52}$. Elle est l'illustration parfaite de cette évolution du cadrage. Fondée en 1993 sous le nom d'Alliance Defense Fund, ce n'est sans doute pas un hasard si elle est rebaptisée en 2012 Alliance Defending Freedom. Comme son pendant catholique, le Becket Fund for Religious Liberty, qui porte le nom d'un martyr anglais du XIIe siècle, elle plaide la cause de la liberté religieuse devant les tribunaux, et a remporté de nombreuses victoires dans les plus hautes cours du pays. Le nouvel 
œcuménisme se manifeste d'ailleurs au prétoire, puisque catholiques et évangéliques interviennent côte à côte dans les affaires alléguant des violations de la liberté religieuse. Ainsi, c'est le catholique Becket Fund qui a défendu l'école luthérienne évangélique Hosanna-Tabor contre la commission fédérale d'égalité d'accès à l'emploi devant la Cour suprême en 2012, laquelle a rendu un arrêt étendant l'exception religieuse aux lois anti-discrimination en matière d'emploi ${ }^{53}$. Les évêques catholiques avaient d'ailleurs déposé un mémoire d'amicus curiae pour appuyer le plaignant ${ }^{54}$. Dans leur comparution devant la Cour suprême pour faire reconnaître l'extension du droit à l'objection de conscience à une entreprise privée, la société mennonite Conestoga fut représentée par l'évangélique $\mathrm{ADF}$, tandis que l'entreprise de loisirs créatifs aux propriétaires évangéliques fut défendue par le catholique Becket Fund.

Les allégations de menaces pesant sur la liberté religieuse sont susceptibles d'avoir un impact plus grand encore sur les droits des femmes et des homosexuels dans la mesure où elles rencontrent un certain écho dans l'opinion publique, comme en témoignent plusieurs sondages faisant état d'une inquiétude quant au déclin de la liberté religieuse dans le pays, ainsi qu'un soutien croissant à des exemptions ${ }^{55}$. S'il demeure difficile de déterminer si ce sentiment de déclin a été alimenté en partie par le cadrage adoptée depuis dix ans par la droite chrétienne, ou si cette dernière a su capter l'humeur de l'opinion, le Pew Research Center a constaté une hausse du pourcentage d'Américains jugeant l'administration Obama hostile à la religion, de $17 \%$ en 2009 à $29 \%$ en 2014, les chiffres pour les protestants évangéliques sur la même période passant de $38 \%$ à $57 \%$, et pour les catholiques blancs de $17 \%$ à $36 \%^{56}$. Entre 2014 et 2016, la part de protestants évangéliques qui estiment qu'il « devient de plus en plus difficile d'être chrétien évangélique aux Etats-Unis » est passée de 34 à $41 \%{ }^{57}$. Interrogés sur ce qu'ils pensaient de l'affirmation « en Amérique aujourd'hui, la discrimination contre les chrétiens est devenue un problème aussi important que la discrimination contre les autres groupes », $49 \%$ ont répondu par la positive, le chiffre s'élevant à $53 \%$ pour les catholiques blancs et $77 \%$ pour les évangéliques blancs ${ }^{58}$.

Le discours des droites chrétiennes sur les menaces que la liberté reproductive et le mariage homosexuel feraient peser sur la liberté religieuse se retrouve aussi chez les membres du Parti républicain avec force depuis les élections de 2012. Le fait pour des candidats et élus républicains de reprendre les préoccupations des droites chrétiennes n'est pas nouveau. On a ainsi pu entendre Richard Nixon faire sienne les préoccupations du cardinal-archevêque de New York sur l'avortement, ou Ronald Reagan reprendre à son compte les revendications de la National Association of Evangelicals à ce même sujet ou sur celui de la prière à l'école. Cependant, ces discours n'ont en général pas donné lieu à des actions concrètes de leur part, les présidents préférant investir leur capital politique ailleurs. Cependant, on constate d'ores et déjà les nombreuses prises de positions lors du débat autour du mandat contraceptif en 2012, le président de la Chambre des Représentants, le catholique John Boehner, qualifiant en pleine séance le mandat d' "attaque univoque sur la liberté religieuse de notre pays ». La sénatrice républicaine, elle aussi catholique, Kelly Ayotte montrait quant à elle qu'elle avait bien intégré la stratégie des droites chrétiennes en déclarant que l'opposition au mandat contraceptif n'avait « rien à voir avec les droits des femmes, mais avec la liberté religieuse $»^{59}$. La campagne pour les présidentielles, la même année, fut l'occasion pour 
les candidats républicains de rivaliser d'indignation quant aux supposées violations, tandis que les lobbies religieux tels que la National Organization for Marriage cherchait à obtenir des candidats qu'ils signent son « engagement pour le mariage » (que paraphèrent Newt Gingrich, Rick Perry, Rick Santorum, Michele Bachmann, et Mitt Romney). Ce texte appelait notamment de ses vœux la formation par le vainqueur des urnes d'une « commission présidentielle sur la liberté religieuse » ${ }^{60}$.

Lors d'un débat entre candidats à la vice-présidence en octobre 2012, le républicain catholique Paul Ryan accusa le président d' « attaquer la liberté religieuse des organisations, hôpitaux et Eglise catholiques ${ }^{61}$, tandis que le candidat à la présidence, le mormon Mitt Romney, alla même jusqu'à déclarer que face aux attaques de l'administration Obama, «nous sommes tous catholiques » ${ }^{62}$. Les primaires républicaines de 2016 sont l'occasion d'un engouement redoublé pour cette thématique de la part des candidats républicains, le favori des électeurs évangéliques, Ted Cruz, se tenant « sans équivoque » aux côtés de la greffière du Kentucky Kim Davis, à laquelle il rendit visite en prison ${ }^{63}$ et de « tous ceux que le gouvernement cherche à persécuter pour avoir défendu leur foi $»{ }^{64}$. Il a également déclaré que dès son premier jour à la Maison-Blanche, il donnerait l'ordre au ministère de la Justice, au fisc et à toutes les agences fédérales que la persécution de la liberté religieuse «s'arrête immédiatement ${ }^{65}$.

De nombreuses églises, ordres religieux et organisations caritatives ont porté plainte contre le mandat contraceptif ; parmi les plaignants figurent l'archidiocèse de New York, l'université fondamentaliste de Jerry Falwell, Liberty University, l'ordre catholique Petites Soeurs des Pauvres ${ }^{66}$, la prestigieuse université Notre-Dame, mais aussi des entreprises privées à but lucratif, comme le groupe de menuiserie Conestoga et la société de fournitures de loisir Hobby Lobby, détenues respectivement par des mennonites et des évangéliques. Tous, y compris les sociétés privées, arguent que leur liberté religieuse est violée par l'obligation qui leur est faite de financer la contraception de leurs salariées. En juin 2014, la Cour suprême fit sienne la nouvelle définition de la liberté religieuse mise en avant par les chrétiens conservateurs, en reconnaissant aux entreprises privées un droit à la liberté religieuse et le droit de s'exonérer de lois qu'ils jugent contraires à leurs convictions religieuses. Cette décision est fondamentale, puisqu'elle étend le droit à l'objection de conscience à une entreprise à but lucratif dont l'activité n'est pas de nature religieuse . En outre, cet arrêt témoigne bien, au-delà de la portée symbolique des propos des uns et des autres sur une nécessaire protection du droit à l'objection de conscience religieusement fondé, de l'impact de cette campagne de cadrage autour de la liberté religieuse d'une minorité chrétienne opprimée. Rappelons au passage les liens étroits entre le Becket Fund et l'USCCB, puisque le personnel passe de l'un à l'autre avec aisance, tel cet ancien avocat du Becket Fund nommé en 2007 à un poste important de juriste à l'USCCB ${ }^{67}$. Cet arrêt est fondé en grande partie sur une loi fédérale de 1993, la Religious Freedom Restoration Act (RFRA), dont l'adoption dans les assemblées des Etats est également devenue un cheval de bataille de la campagne pour la défense religieuse.

Le pouvoir législatif est aussi devenu une des cibles des conservateurs chrétiens. Leur action porte d'une part sur l'échelon fédéral, les évêques catholiques et les responsables baptistes du sud soutenant en juin 2015 un projet de loi connu sous le nom de Loi de défense du Premier Amendement (First Amendment Defense Act, ou FADA), qui empêcherait le gouvernement fédéral de révoquer l'exonération fiscale des Eglises en cas de 
décision considérée comme discriminatoire par certaines lois ou de pénaliser les organisations religieuses telles des écoles pour avoir refusé des enfants de couples de même sexe $^{68}$ ou, en avril 2015, le catholique Mgr Lori et le baptiste du Sud Russell Moore écrivant conjointement aux membres du Congrès pour les exhorter à voter une loi, la Health Care Conscience Rights Act, pour renforcer les protections à l'objection de conscience dans la santé ${ }^{69}$.

Les mouvements religieux conservateurs sont également très actifs auprès des assemblées des Etats. On assiste à une vaste mobilisation pour faire adopter des lois RFRA dans les Etats. Adoptée en 1993 du temps de Bill Clinton à la quasi-unanimité et soutenue aussi bien par des organisations progressistes que conservatrices, la loi RFRA avait pour but de rétablir la protection de la pratique religieuse, que la Cour suprême avait restreinte par l'arrêt Employment Division v. Smith de 1990 évoqué supra. Cette loi visait essentiellement à protéger le libre exercice de sa religion pour des confessions minoritaires. La Cour suprême avait invalidé en 1997 la provision de la loi qui imposait aux Etats fédérés et non seulement à l'Etat fédéral d'adopter la lecture la plus large possible du libre exercice de la religion, ce qui avait suscité l'adoption immédiate par plusieurs Etats de leur propre RFRA, là encore avec le soutien de groupes de tous bords politiques ${ }^{70}$. Depuis 2013 en revanche, on assiste à une « deuxième vague » de lois RFRA (Kansas, Kentucky, Mississippi, Ohio, Arizona, Indiana, Oklahoma... ), vague qui s'est accélérée après l'arrêt Hobby Lobby de juin 2014. Ces lois RFRAs, qui ont souvent déclenché l'indignation, présentent une différence significative par rapport à celles de la première vague, puisqu'elles comportent des dispositions garantissant la plus grande liberté possible dans l'exercice de sa religion non seulement aux Eglises, mais aussi aux individus et aux entreprises à but lucratif, ce qui revient à permettre aux entreprises de quelque nature qu'elles soient à se soustraire aux lois anti-discriminations, notamment vis-à-vis des femmes et des homosexuels ${ }^{71}$. Par ailleurs, les lois RFRA originelles visaient exclusivement à imposer ce critère de liberté très vaste dans les relations entre le gouvernement et des individus ou des entités religieuses, mais les nouvelles moutures s'appliquent également aux relations entre entités de droit privé ${ }^{72}$. Encore une fois, on observe la logique d'inversion entre minorité et majorité, puisque les catholiques et évangéliques utilisent ces lois jadis utilisées pour les minorités religieuses en s'appuyant sur des allégations de persécution à leur encontre. Derrière la présentation de ces projets de loi, on voit l'influence de lobbies religieux comme le Becket Fund, l'Alliance Defending Freedom, d'Eglises comme les Eglises catholique ou mormone, qui ont conseillé les législateurs de ces Etats ${ }^{73}$.

Toutes les plaintes contre le mandat contraceptif ont utilisé les dispositions ultraprotectives de la loi fédérale RFRA pour se justifier. Et elles ont eu gain de cause auprès de la Cour, après le résultat précoce d'une autre plainte, plus rapidement traitée, celle de l'entreprise Hobby Lobby qui refusait - toute comme l'entreprise de menuiserie Conestoga - d'appliquer elle-aussi le mandat. En 2014, l'arrêt Hobby Lobby v. Sebelius ${ }^{74}$ est rendu par la Cour suprême. Outre que cette dernière entérine de manière très contestable le droit des entreprises privées à but lucratif à bénéficier des dispositions du RFRA, la Cour accepte l'argument de l'entrave à la liberté religieuse qui est avancé par cette entreprise - et par la suite à tous les autres types de plaignants concernant le mandat - sans questionner la notion de fardeau substantiel ou de contrainte insupportable posé dans le RFRA. Dans la doctrine majoritaire de la Cour, le contenu subjectif de 
l'exercice de la religion - comme absolu du for interne extérieurement limitable selon la jurisprudence fondatrice du cas Reynolds - est devenu à la fois insaisissable et prioritaire.

\section{Les effets de la lutte jurisprudentielle contre le contraceptive mandate}

La lutte jurisprudentielle sur le mandat contraceptif, lancée par les organisations catholiques et aboutie dans l'affaire Hobby Lobby a produit en outre trois effets boule de neige :

- Tout d'abord, la montée des plaintes pour refus de complicité de péché ${ }^{75}$ dans le cas du mandat et bientôt dans le cas de lois d'Etat et ou de jurisprudences fédérées protégeant l'homosexualité comme un droit égal et le mariage civil homosexuel comme un mariage « égal».

- Ensuite l'utilisation de la logique du RFRA pour fabriquer dans les Etats des clones du RFRA ou mini-RFRA, permettant d'attaquer frontalement les lois des Etats autorisant les mariages homosexuels et l'avortement précoce qui sont réglés à leur niveau.

- Enfin, la mise en route par le Congrès républicain sous Barack Obama de deux projets de loi quasi aboutis, le Health Care Conscience Rights Act et le First Amendment Defense Act, consécration de l'objection et de la discrimination religieuses actives.

Commençons par le refus de complicité de péché comme angle d'attaque judiciaire. Il s'agit d'une catégorie de revendications faites par des individus ou des groupes qui refusent de se conformer aux lois communes, non parce qu'elles les empêchent d'exercer une quelconque obligation religieuse positive, mais parce que cette adhésion les rendrait complices des péchés des autres. L'idée elle-même n'est pas nouvelle aux États-Unis, ni propre d'ailleurs à la culture américaine : dans les années 1980, le mouvement de lutte contre la ségrégation raciale et les décisions de sanctions contre l'Afrique du Sud sont un exemple fort de l'argument de refus de complicité, qui a recueilli un soutien très large aux États-Unis et dans la communauté internationale. Cependant, les cas qui se sont posés depuis 2012 sont fondés, comme nous l'avons vu, sur l'idée qu'on ne doit pas être complice du péché d'autrui.

Dans plusieurs affaires récemment jugées, les plaignants ont rejeté le compromis proposé par la Cour dans l'affaire des Petites Sœurs des Pauvres: être autorisés à déposer un formulaire notifiant l'objection de l'assureur au paiement de la couverture contraceptive. Ces plaignants ont insisté sur le fait qu'une telle notification ne les sauvait pas de cette complicité au péché d'autrui, puisque l'avis permettait la contraception par d'autres moyens. Bien que les tribunaux inférieurs aient rejeté ces allégations, la Cour suprême a récemment renvoyé auprès de ces tribunaux plusieurs cas similaires, pour trouver de nouveaux compromis ${ }^{76}$.

Avec la légalisation du mariage homosexuel dans une quinzaine d'États depuis 2011, les refus de complicité ont commencé à se multiplier, dans lesquels les entreprises ou 
artisans refusent de fournir leurs services aux couples homosexuels, arguant qu'ils avaient le droit de discriminer les homosexuels pour ne pas être complices de leur péché, comme dans le cas dans l'affaire Elane Photography ${ }^{77}$ de 2013 , où un photographe professionnel a refusé de photographier une célébration de mariage homosexuel, au motif de sa désapprobation spirituelle. Une autre affaire a impliqué un fleuriste, et une autre encore un boulanger chrétien qui a refusé de créer un gâteau de mariage pour un couple de même sexe $\mathrm{e}^{78}$. Certains cas sont presque drôles : un client chrétien commande à une boulangerie gay-friendly de préparer des gâteaux avec des messages tels que «l'homosexualité est un péché détestable » ${ }^{79}$. Les tribunaux ont soutenu jusqu'à présent le droit du gouvernement à adopter et à faire respecter les lois antidiscriminatoires.

Deuxièmement, la montée en puissance de l'exemption religieuse aux lois générales, au nom d'une liberté religieuse contrainte ou blessée ou d'une conscience religieuse attentive à ne pas pêcher, a eu un autre effet d'entraînement. Ceux qui brandissent le droit à la liberté religieuse ont été encouragés après la décision Hobby Lobby à militer pour obtenir des Chambres l'adoption de variantes du RFRA au niveau des États - des mini-RFRA - convaincus que ces lois leur donneraient de solides moyens contre le mariage homosexuel civil, indirectement admis partout par la jurisprudence fédérale Obergefell v. Hodges de $2015^{80}$, ou encore contre l'avortement précoce, encadré par plus de 500 lois au niveau des États eux-mêmes. Depuis 2014, 22 États ont ainsi introduit des mini-RFRA ou mis en chantier la modification de leur loi RFRA quand elle existait, dont certaines incluent explicitement les sociétés, comme l'Oklahoma, quand les autres conservent, sans loi complémentaire, l'interprétation judiciaire fédérale de l'arrêt Employment Division v. Smith. Derrière la présentation de ces projets de loi, on voit l'influence de lobbies religieux comme le Becket Fund, l'Alliance for Defending Freedom et d'Eglises comme les Eglises catholique ou mormone, qui ont conseillé les législateurs de ces États. Ces récentes tentatives de durcir la protection de la liberté religieuse par les mini-RFRAs ont été âprement combattues. Seuls l'Indiana, l'Arkansas et la Virginie ont réussi à adopter une loi ou des amendements proches du RFRA - signé en Indiana par le gouverneur de l'Etat Mike Pence - et ils ont eu à faire face à une résistance très vive. Des manifestants ont tenté en mars 2015 de s'opposer au passage de l'Indiana Religious Freedom Restoration Act, faisant écho à des manifestations similaires en Géorgie, dans l'Arkansas, le Colorado ou encore le Michigan, au nom de la prévisible discrimination dont allaient être victimes les homosexuels. En effet ces États - et ils sont majoritaires n'ont pas de disposition qui interdise la discrimination pour orientation sexuelle. Si l'Indiana a finalement inclus une clause interdisant aux entreprises de refuser leurs services ou leurs biens à un citoyen en vertu de leurs convictions religieuses blessées, d'autres législatures comme celle de l'Oklahoma ont des projets de textes en discussion qui autorisent directement des individus et/ou des entités religieuses à avoir un droit de réserve religieuse, ce qui signifie concrètement qu'ils peuvent refuser leur service à des personnes, refuser d'embaucher du personnel ou licencier pour raison religieuse. ${ }^{81}$

Enfin, apparente consécration de ce mouvement de surprotection de la conscience religieuse, les projets de loi fédérale, Health Care Conscience Rights et First Amendment Defense Act introduits à la Chambre des Représentants et au Sénat par des Républicains. Le premier en mars 2013 interdit toute sanction financière- comme le refus de subventions publiques- contre les organismes d'assurances privées qui refusent d'inclure les 
frais d'avortement dans leur plan de santé. Le deuxième, en juin 2015, projette d'interdire au gouvernement fédéral toute action discriminante contre toute personne ou organisations « qui croit ou agit en accord avec sa croyance religieuse ou sa conviction morale que le mariage est ou doit être reconnu comme l'union d'un homme et d'une femme et que les relations sexuelles sont réservés à un tel mariage ${ }^{82}$. Les nombreux instigateurs derrière ces deux projets sont les lobbies chrétiens spécialisés comme le Family Research Council, ou 1'American Family Association de tempérament fondamentaliste. Ils ont reçu le ferme soutien public de ''USCCB $^{83}$, par la voix du cardinal O’Malley de Boston, puis celle de Mgr Salvatore Cordileone, archevêque de San Francisco, successivement présidents du comité de la conférence pour la défense de la famille et du mariage, et par celle de Mgr William Lori, toujours président du comité ad hoc pour la liberté religieuse.

Dans leur soutien public, les évêques insistent sur la protection apportée par le deuxième projet de loi, pour empêcher l'Etat de retirer les contrats publics aux organisations refusant le mandat contraceptif ou le mariage homosexuel ou le droit à l'avortement - mettant à mal le décret de Barack Obama interdisant toute forme de discrimination sexuelle ou de genre pour les organisations et entreprises travaillant avec le gouvernement et les services fédéraux- même chose pour l'octroi des subventions publiques, le statut d'exempté fiscal ou l'embauche discriminée au sein de ces organisations $^{84}$. Le projet protège l'exonération fiscale des Églises en cas de décision jugée discriminatoire par certaines lois ou empêcherait de pénaliser les organisations religieuses telles des écoles pour avoir refusé des enfants de couples de même sexe. Depuis 2015, 15 Etats ont également introduit des projets de mini-FADA équivalents. Les gouverneurs de Géorgie et d'Arizona ont bloqué les lois qui ont été votées. Ailleurs, sous la pression d'organisations protégeant les droits des homosexuels ou luttant contre les discriminations de genre, mais aussi la pression de grands groupes comme Apple (en Indiana), Dell ou Microsoft, les lois n'ont finalement pas été votées ou elles ont été fortement édulcorées.

\section{« La fin de la discrimination des croyants » : la politique publique religieuse du Président Trump}

Autre signe du succès de cette stratégie, l'adoption de ce discours par Donald Trump. Dès la campagne présidentielle, le candidat républicain avait fait sienne la rhétorique de menace sur la liberté religieuse des chrétiens opprimés. Il évoqua à l'envi le sort des Petites Sœurs des Pauvres et promit aux catholiques américains qu'il « serait là pour eux », qu'il se « tiendrait à leurs côtés et qu'il se « battrait pour eux » dans une lettre adressée à la communauté catholique en octobre 2016. Il y évoquait également le mandat contraceptif pour signaler qu'ils ne verraient jamais «ce genre d'hostilité à la liberté religieuse $[\ldots]$ sous une présidence Trump $»{ }^{85}$. Il avait déclaré en janvier 2016 dans une université chrétienne que le christianisme est « assiégé de partout » ${ }^{86}$ et s'était engagé à encourager la nouvelle présentation au Congrès du projet de loi FADA ${ }^{87}$.

Depuis son entrée en fonction, l'administration Trump a adopté plusieurs mesures visant à protéger la liberté religieuse. En mai 2017, le Président a signé à grand renfort de personnalités religieuses supposément atteintes par ces violations - dont les Petites Sœurs des Pauvres - et de propos grandiloquents sur sa volonté de « ne plus laisser les 
croyants être pris pour cible, harcelés et muselés » le décret «Promoting Free Speech and Religious Liberty ». Pour Trump, « pendant trop longtemps, le gouvernement fédéral a utilisé le pouvoir de l'Etat comme arme contre les croyants, brutalisant et même punissant les Américains pour avoir suivi leurs croyances religieuses ». Désormais, leur annonce-t-il, « leur calvaire est terminé ${ }^{88}$. Le décret comporte trois volets. Il consiste tout d'abord à promettre que l'exécutif fera respecter avec force les systèmes de protection de la liberté religieuse ; il annonce ensuite que le département du Trésor ne prendra pas de mesures de rétorsion contre les individus ou organisations religieuses ayant parlé de questions morales d'un point de vue religieux. Enfin, il annonce que les ministères du Trésor, du Travail et de la Santé sont appelés à édicter des normes pour répondre aux objections opposées au mandat contraceptif ${ }^{89}$.

Annoncé en grande fanfare comme la fin de la discrimination des croyants aux EtatsUnis, ce décret a néanmoins reçu un accueil mitigé de la part des chrétiens évangéliques, dans la mesure tout d'abord où la possibilité de montrer son soutien pour tel ou tel candidat importe peu aux évangéliques ${ }^{90}$. Il est en outre assez peu ambitieux, notamment en ce qui concerne la protection qu'il offre aux personnes dont les convictions religieuses se heurtent aux lois anti-discrimination contre les Américains LGBT par rapport au projet de décret qui avait fuité dans la presse en février ${ }^{91}$. En outre, les évangéliques se plaignent du fait que toute mesure mise en œuvre par le biais d'un décret présidentiel peut être abrogée par le président suivant, et qu'il est impératif que des lois soient votées en ce sens ${ }^{92}$. La Conférence des évêques avait exhorté le nouveau président à agir en faveur de la liberté religieuse ${ }^{93}$ et se réjouit publiquement du décret présidentiel du 5 mai 2017 sur la liberté religieuse. Cependant le décret s'est révélé à ce point sans consistance - si ce n'est de permettre aux organisations religieuses de dépenser leur argent en faveur de partis politiques - que l'ACLU a renoncé à l'attaquer en justice.

En octobre 2017, deux nouvelles directives sont rendues publiques. La première émane du ministre de la Justice Jeff Sessions, connu pour son scepticisme quant à la pertinence de la séparation entre les Eglises et 1'Etat. Elle donne une interprétation très large de la liberté religieuse, réinterprétant celle édictée par la loi RFRA de 1993 et lui substituant celle promue par les tenants de la droite chrétienne, selon laquelle la liberté religieuse ne doit pas être comprise comme un simple droit personnel de culte dans un lieu sacré, mais doit dans la limite du raisonnable et de la législation faire l'objet d'accommodements les plus larges possibles dans toutes les activités gouvernementales, que ce soir en matière d'emploi, de sous-traitance ou de programme ce qui permettrait par exemple à une entreprise sous-contrat avec l'Etat fédéral de ne pas avoir à respecter les lois anti-discrimination au nom de leurs croyances. L'autre directive émane du ministère de la Santé : elle abroge de facto le mandat contraceptif en donnant à tout employeur, qu'il soit un individu ou une société cotée en bourse le droit de s'en affranchir au nom d'une « croyance religieuses sincère » ou de ses « convictions morales » qui le conduisent à ne pas vouloir financer la contraception de ses salariés.

Ces mesures sont le fruit de nominations faites par Trump. C'est d'ailleurs peut-être ces dernières qui constituent la plus grande avancée pour les catholiques et évangéliques. On sait que l'un des principaux auteurs des directives évoquées supra est Matthew Bowman, un des juristes les plus haut-placés au ministère de la Santé, et un ancien de l'Alliance Defending Freeedom. La décision de supprimer le mandat a été prise juste 
après sa nomination ${ }^{94}$. A la division des droits civiques du ministère a été nommé un ancien du Becket Fund, Roger Severino, qui en janvier 2018 est placée à la tête d'une nouvelle division «Conscience et Liberté religieuse », dont le rôle est d'enquêter sur les plaintes déposées par des personnels de santé qui auraient été contraints d'accomplir des actes contraires à leurs convictions religieuses ou morales ${ }^{95}$. La création de cette division et la norme qui l'accompagne constituent l'abrogation du décret d'Obama de 2011 limitant les cas de recours à l'objection de conscience pour motif religieux, et certains craignent de ce fait un retour aux discriminations contre les femmes, les homosexuels ou les transgenres dans l'accès aux soins.

Dernier gage, et non des moindres, donné à l'électorat conservateur chrétien, les nominations judiciaires. Le président Trump a pu nommer deux juges chrétiens conservateurs, Neil Gorsuch et Brett Kavanaugh, dont la présence à la Cour suprême est une garantie supplémentaire que cette défense de la liberté religieuse, même si elle est synonyme de discrimination envers les femmes ou les personnes transgenres ou homosexuelles, sera jugée constitutionnelle. Mais les nominations clés ne s'arrêtent pas à cet échelon très médiatique du système judiciaire américain, puisque le Président a déjà nommé dans des cours fédérales de niveau inférieur (circuit ou appel) un nombre importants de juges, dont le mandat est aussi dépourvu de limite temporelle. En moins d'un an après son investiture, il avait déjà nommé quatre juges fédéraux ayant des liens étroits avec l'Alliance Defending Freedom ${ }^{96}$. Il a également nommé des juges liés au Becket Fund, dont Amy Conney Barrett, un temps pressentie pour succéder à Anthony Kennedy à la Cour suprême, ou Kyle Duncan ${ }^{97}$.

\section{Conclusion}

Les chances pour que le FADA soit finalement voté par le Congrès et signé par le Président, avaient augmenté depuis l'arrivée de Donald Trump à la Maison Blanche. En mars 2018, le sénateur Mike Lee l'a réintroduit auprès du Sénat avec le soutien de 21 sénateurs républicains, mais le changement de majorité à la Chambre des Représentants à l'issue des élections de 2018 augurent mal de son adoption. De même, le mandat contraceptif était inclus dans le démantèlement de l'Affordable Care Act, mais celuici n'a finalement pas eu lieu, grâce au vote historique du sénateur républicain John McCain et des sénatrices républicaines Lisa Murkowski et Susan Collins contre son abrogation en juillet 2017. La Cour suprême, du fait des nouvelles nominations a cependant de grandes chances de poursuivre son interprétation extensive du RFRA. Par ailleurs, les moyens légaux dont ont cherché à se doter les Etats par le biais des mini-RFRA et des mini-FADA, même réduits à d'autres tentatives plus modestes, vont se fonder sur le droit d'objection et de discrimination religieuse comme nouvelle interprétation de la liberté religieuse atteinte par les législations sur l'avortement, le mariage homosexuel et la santé reproductive. Dans cette bataille ciblée, la Conférence des évêques joue la même partition que les grandes organisations évangéliques et les lobbies politiques de la droite religieuse.

Le recours à une approche axée toute entière sur la défense de la liberté religieuse comme résistance à l'oppression de la loi sans foi en matière de santé reproductrice ou de reconnaissance des droits homosexuels, présente de nombreux avantages. Elle permet 
d'éviter l'inaudibilité de la critique morale de la contraception ${ }^{98}$ dans un pays où sa pratique est très élevée et son usage considéré comme moralement neutre. Elle permet de cibler simultanément des publics assez disparates, dont certains n'étaient jusqu'à présent pas très sensibles à ces préoccupations. Outre les chrétiens conservateurs, cœur de cible traditionnel, cette approche est susceptible de rencontrer un écho fort chez les libertariens, favorables à un rôle réduit de l'État dans la société, et chez les modérés, qui peuvent être sensibles à l'argument d'une menace sur les droits civiques. La révolte morale contre la violation de la liberté religieuse est devenu un argument rassembleur, comme le prouve la déclaration du directeur de la commission Éthique et Liberté religieuse de la plus grande confession protestante du pays, la Southern Baptist Convention : «l'enjeu ici n'est pas l'avortement $[\ldots]$ mais le mépris et l'insensibilisé dont a fait preuve notre gouvernement vis-à-vis de la liberté des Américains à appliquer leurs convictions religieuses $»^{99}$. Grâce à cette posture « indignée », appelant d'abord à la désobéissance civile puis à l'intégration légale de l'objection de conscience religieuse en cas de conflit de loyauté avec les lois existantes, le camp militant de l'Amérique chrétienne a trouvé un coin prometteur pour enfoncer son clou convictionnel, bien au-delà de la question du contraceptive mandate.

Ces mesures feront fort probablement l'objet de procès, or c'est là que les nominations judiciaires de l'administration prennent tout leur poids. En nommant comme il l'a fait deux conservateurs sur les questions de «liberté religieuse » et d'avortement, en nommant des juges fédéraux au diapason de ses juges à la Cour suprême, juristes qui ont fait leurs armes pour certains d'entre eux dans les rangs du Becket Fund ou de l'Alliance Defending Freedom, Trump entend donner à la Cour suprême l'occasion de pérenniser en faisant d'elles par un arrêt de la jurisprudence ces mesures mises en œuvre par la voix du décret ou de la directive, et de ce fait susceptibles d'être abrogées par un futur président démocrate.

Le cadrage axé autour de la défense de la liberté religieuse semble donc pour l'instant un choix plutôt judicieux de la part de l'USCCB et des chrétiens conservateurs. A la grand-messe annuelle de la Faith and Freedom Coalition de Ralph Reed, l'un des fondateurs de la droite chrétienne et l'un des premiers à prôner ce cadrage minoritaire, Trump a ainsi promis à son auditoire de «mettre un terme à la discrimination contre les croyants» et de «célébrer et protéger à nouveau la liberté religieuse ${ }^{100}$. Cet événement, qui se tient depuis 2009 , période à partir de laquelle le recours au vocable de la liberté religieuse se fait massif chez les chrétiens conservateurs, porte le nom de « Road to Majority », « en route pour la majorité » : que ce soit en se présentant comme une majorité dominante ou une minorité opprimée, l'objectif demeure bien d'imposer par le biais de la loi et de la jurisprudence les valeurs chrétiennes sur la famille et le mariage à l'ensemble de la société américaine.

\section{Déclaration de conflits d'intérêts}

L'auteur déclare qu'il n'y a aucun conflit d'intérêt à l'égard de la recherche, les droits d'auteurs et / ou la publication de cet article. 


\section{Financement}

L'auteur n'a pas reçu de soutien financier pour la recherche, les droits d'auteurs et / ou la publication de cet article.

\section{Notes}

1. McCarthy J (1997) The Globalization of Social Movement Theory. In: Smith J, Chatfield C et Pagnucco R (eds) Transnational Social Movements and Global Politics: Solidarity beyond the State. Syracuse, NY: Syracuse University Press, p. 244.

2. Miceli M (2005) Morality Politics vs. Identity Politics: Framing Processes and Competitions among Christian Right and Gay Social Movement Organizations. Sociological Forum 20(4) : $590-595$.

3. Wilcox C et Robinson C (2010) Onward Christian Soldiers? The Religious Right in American Politics. Boulder, CO: Westview Press.

4. Diamond S (2000) Not by Politics Alone. The Enduring Influence of the Christian Right. New York: The Guilford Press.

5. Marley DJ (2006) Riding in the Back of the Bus. The Christian Right's Adoption of Civil Rights Movement Rhetoric. In: Romano R et Raiford L (eds) The Civil Rights Movement in American Memory. Athens: The University of Georgia Press, p. 347. Voir également Haberman AL (2006) Civil Rights on the Right: The Modern Christian Right and the Crusade for School Prayer 1962-1996. Thèse de doctorat, University of South Carolina.

6. Ibid., pp. 346-348.

7. Ibid., pp. 347-348.

8. Klemp N (2012) The Morality of Spin. Virtue and Vice in Political Rhetoric and the Christian Right. Lanham, MD: Rowman and Littlefield, pp. 92-97. Miceli M, op. cit, pp. 589-612.

9. Saad L (2012) US Acceptance of Gay/Lesbian Relations is the New Normal. Gallup, 12 mai. http://www.gallup.com/poll/154634/acceptance-gay-lesbian-relations-new-normal.aspx.

10. Newport F (2012) Half of Americans Support Gay Marriage. Gallup, 8 mai. http://www. gallup.com/poll/154529/half-americans-support-legal-gay-marriage.aspx, PEW (2013) Growing Support for Gay Marriage: Changed Minds and Changed Demographics. Pew Research Center, 20 mars. http://www.people-press.org/files/legacy-pdf/3-20-13 \%20Gay\%20Marriage\%20Release.pdf.

11. Newport F (2012). Americans, Including Catholics, Say Birth Control is Morally OK. Gallup, 22 mai. http://www.gallup.com/poll/154799/americans-including-catholics-say-birth-controlmorally.aspx.

12. PEW (2012) People Divided over Birth Control Insurance Mandate. 14 février. http://www. people-press.org/2012/02/14/public-divided-over-birth-control-insurance-mandate/.

13. PEW (2015) America's Changing Religious Landscape. 12 mai. http://www.pewforum.org/ 2015/05/12/americas-changing-religious-landscape/.

14. MARLEY, op. cit., pp.348-349.

15. Ibid, p. 352.

16. Ibid., p.352.

17. Lyons L (2002) The Gallup Brain: Prayer in Public Schools. Gallup, 10 décembre. http:// www.gallup.com/poll/7393/gallup-brain-prayer-public-schools.aspx.

18. Klemp, op. cit., p. 91. 
19. Stone A (2012) The New Religious Freedom Argument? The Public Eye 27(3): 11-14.

20. Audi T et Gershman J (2015) Religious Groups Vow to Fight Gay Marriage despite Supreme Court. Wall Street Journal, 16 juin. http:/www.wsj.com/articles/religious-groups-vow-tofight-same-sex-marriage-despite-supreme-court-61435329751. Posner S (2012) In 2012 Bishops join effort to repackage discrimination as 'religious freedom. Religion Dispatches, 13 mars. http://religiondispatches.org/in-2012-bishops-join-fight-to-repackage-discrimina tion-as-religious-freedom/.

21. Michaelson J (2016) Redefining Religious Liberty: The Covert Campaign against Civil Rights. Political Research, 20 mars. https://www.politicalresearch.org/2013/03/21/redefin ing-religious-liberty-the-covert-campaign-against-civil-rights/.

22. Laura R (2013) Pastor Rick Warren Is Well-Prepared for a Purpose-Driven Retirement. Forbes, 21 mars. https://www.forbes.com/sites/robertlaura/2013/03/21/pastor-rick-warren-ispracticing-what-he-preaches-and-getting-ready-for-retirement/\#56630d584dbf;Michaelso n,op.cit.

23. Weigel D et Delreal J (2015) Kim Davis accepts award for denying marriage licenses to gays. Washington Post, 25 septembre, https:/www.washingtonpost.com/news/post-politics/wp/ 2015/09/25/kim-davis-has-her-moment/.

24. Hausknecht B (2013) Religious Freedom in Danger. Voir : https://www.focusonthefamily. $\mathrm{com} /$ socialissues/religious-freedom/religious-freedom-in-danger/religious-freedom-indanger.

25. Stein R (2011) Obama Administration Replaces Controversial 'Conscience' Regulation for Health-Care Workers. Washington Post, 19 février, https://www.washingtonpost.com/ national/health-conscience-rule-replaced/2011/02/18/AB7s9iH_story.html?utm_term =. $8904 \mathrm{f5a} 4 \mathrm{~b} 8 \mathrm{a} 6$.

26. Goodstein L (2011) Bishops Open Religious Liberty Drive. New York Times, 14 novembre http://www.nytimes.com/2011/11/15/us/bishops-renew-fight-on-abortion-and-gay-marriage. $\mathrm{html}$ ? $\mathrm{r}=0$.

27. USCCB, Religious Liberty at Home. http://www.usccb.org/issues-and-action/religious-lib erty/religious-liberty-at-home.cfm.

28. Cités in Gayte M (2016).

29. Condamnation en 2012 pour « sape et dissidence » de la foi catholique.

30. Berry J (2012) A New Inquisition? The Vatican Targets American Nuns. National Catholic Reporter, 26 décembre. http://ncronline.org/news/sisters-stories/new-inquisition-vatican-tar gets-us-nuns.

31. Enda J (2012) US Bishops' Roman Connection. Conscience 33(3): 14-18.

32. Peoria Bishop's Stalin, Hitler References in Homily Stir Controversy (2012). Catholic News Services, 23 avril. http://www.catholicnews.com/data/stories/cns/1201654.htm. Brachear M (2012) Cardinal Writes To Priests Prior To Election. Chicago Tribune, 2 novembre. http:// articles.chicagotribune.com/2012-11-02/news/chi-cardinal-writes-to-priests-prior-to-elec tion-20121102-1-priests-religious-freedom-parish-bulletins.

33. Kaleem J (2012) Cardinal Dolan's Benedictions at RNC and DNC. Huffington Post, 6 septembre, http://www.huffingtonpost.com/2012/09/06/cardinal-dolan-benediction-rnc-dnc-n1861440.html.

34. Le jugement de la Cour Suprême, rendu pour les Little Sisters of the Poor v. Sebelius et six autres cas consolidés ensemble en novembre 2015 - Zubick v. Burwell, Geneva College v 
Burwell, East Texas Baptist University v; Burwell, Southern Nazareen University v. Sebelius, Roman Catholic Archbishop v; Burwell - sera rendu le 16 mai 2016 ( 578 U.S _ (2016), donnant raison aux plaignantes.

35. Reynolds v. United States, 98 U.S. 145 (1878).

36. Ross Sammon M (2008) The Politics of the US Catholic Bishops: The Centrality of Abortion. In: Heyer K, Rozell M and Genovese M (eds) Catholics and Politics. The Dynamic Tension between Faith and Power. Washington DC: Georgetown University Press, pp. 11-26.

37. Living the Gospel of Life, A Challenge for American Catholics (1998). http://www.usccb.org/ issues-and-action/human-life-and-dignity/abortion/living-the-gospel-of-life.cfm : "opposition to abortion and euthanasia is foundational for Catholics and that Catholics should weigh candidates' positions on abortion above other social justice issues such as poverty and war". Sur ces questions, voir Byrnes T (1991) Catholic Bishops in American Politics. Princeton, NJ: Princeton University Press. Ross Sammon M (2008) The Politics of the US Catholic Bishops: The Centrality of Abortion. In: Heyer K, Rozell M et Genovese M (eds) Catholics and Politics. The Dynamic Tension between Faith and Power. Washington DC: Georgetown University Press. Miller P (2014) Good Catholic: The Battle over Abortion in the Catholic Church. Berkeley: University of California Press, p. 106; Sullivan A (2014) The Party Faithful: How and Why Democrats Are Closing the God Gap. New York: Scribner.

Sullivan, op. cit. p. 71. D'Antonio WV (2012) Catholic Bishops and the Electoral Process in American Politics, in Cafardi, op. cit., p. 56. Voir aussi Gayte (2018) The US Catholic Bishops: From Separatism to Interventionism. In: Gayte, Chelini-Pont B et Rozell M (eds) Catholics and US Politics After the 2016 Elections Understanding the 'Swing Vote'. New York: Palgrave Macmillan.

38. Marlin GJ (2004) The American Catholic Voter. 200 Years of Political Impact. South Bend: St Augustine's Press, p. 340.

39. Swole Cahill L (2012) Voting and Living the Common Good. In: Cafardi, op. cit., pp. 27-29.

40. Goodstein L (2013) Pope Says Church Is 'Obsessed' With Gays, Abortion and Birth Control. New York Times, 19 septembre. http://www.nytimes.com/2013/09/20/world/europe/popebluntly-faults-churchs-focus-on-gays-and-abortion.html?pagewanted=all.

41. O’Loughlin M (2015) US Bishops Press On With Marriage, Religious Freedom Goals. Crux, 18 novembre. https://cruxnow.com/church/2015/11/18/us-bishops-press-on-with-marriagereligious-freedom-goals/.

Pour autant, les études montrent que la parole publique des évêques n'est pas systématiquement prise en compte ni connue ni appréciée des électeurs catholiques. cf Smith GA (2008) One Church, Many Messages. In Heyer K, Rozell M and Genovese M (eds) Catholics and Politics. The Dynamic Tension between Faith and Power. Washington DC: Georgetown University Press, pp. 43-60.

42. http://www.usccb.org/news/2004/04-134.cfm.

43. Employment Division, Department of Human Resources of Oregon v. Smith, 494 U.S. 872 (1990). Dans les années 1970 l'épiscopat américain s'est battu pour obtenir un amendement fédéral sur le caractère sacré et inaliénable de la vie, qu'il n'a jamais pu faire aboutir.

44. Au début des années soixante-dix, l'Etat du Wisconsin avait incriminé des parents amish qui avaient refusé d'envoyer leurs enfants à l'école après la classe de quatrième. La Cour suprême donne tort à l'État du Wisconsin, dans Wisconsin v. Yoder, 406 U.S. 205 (1972). Le contrôle de l'ingérence admissible de l'État en matière de liberté religieuse est redéfini : il ne peut être 
porté l'atteinte à la liberté religieuse d'une personne qu'en présence d'un intérêt public impératif et qu'à la condition que l'atteinte en cause soit le moyen le moins lourd pour atteindre l'intérêt impératif en question.

45. La Cour suprême dans l'affaire City of Boerne v. Flores, 521 U.S. 507 (1997), déclare la loi fédérale RFRA inconstitutionnelle pour les États fédérés, car le $14^{\mathrm{e}}$ amendement fédéral (sur laquelle la loi fédérale repose) ne donne pas au Congrès le pouvoir d'imposer aux États des modifications dans l'interprétation de cet amendement. Dans l'arrêt de 2006, Gonzales v. O Centro Espirita Beneficente Uniao do Vegetal, la Cour suprême confirme implicitement la validité du RFRA au niveau fédéral. Pour les États, les lois qui peuvent être en conflit avec le Premier Amendement sont jugées en vertu de la norme simple établie par la Cour dans Smith, qui permet au gouvernement de restreindre la liberté religieuse tant qu'il a un but raisonnable et non discriminatoire et qu'il y a une relation rationnelle entre la loi et le but que le gouvernement espère atteindre.

46. Voir à ce sujet l'ouvrage: Young N (2015) We Gather Together: The Religious Right and the Problem of Interfaith Politics. New York: Oxford University Press. Wilcox C, Merolla LM et Beer D (2006), Saving Marriage by Banning Marriage: The Christian Right Finds a New Issue in 2004. In: Green J, Rozell M et Wilcox C (eds) The Values Campaign?: The Christian Right and the 2004 Elections. Washington DC: Georgetown University Press, p. 58, Berkowitz B (2013) Conservative Catholics and Religious Right Unite for Revamped Culture War. Truthout, 16 juillet. http://truth-out.org/buzzflash/commentary/conservative-catholics-religiousright-unite/18110-conservative-catholics-religious-right-unite, Clarkson F (2013) Christian Right seeks renewal in deepening Catholic-Protestant alliance. Political Research, 23 juillet. http://www.politicalresearch.org/2013/07/23/christian-right-seeks-renewal-in-deepening-cath olic-protestant-alliance/\#sthash.oQPLMemB.6fll7Iv0.dpuf.

47. Manhattan Declaration: A Call For Christian Conscience (2009), 20 novembre. http://manhat tandeclaration.org/man_dec_resources/Manhattan_Declaration_full_text.pdf

48. Clarkson F (2015) Christian Right Leaders Escalate Anti-LGBTQ Threats. Political Research, 4 mai. https://www.politicalresearch.org/2015/05/04/christian-right-leaders-escalate-antilgbtq-threats/.

49. Defend Marriage. Key Signers of the Pledge. http://defendmarriage.org/signers.

50. Speciale A (2012) Benedict gives Directions to US Bishops on Hot-Button Issues. Religion News Service, 22 mai. http://www.religionnews.com/2012/05/22/pope-benedict-xvi-givesdirection-to-u-s-bishops-on-hot-button-issues/.

51. Clermont B (2014) Duped by the Media on Pope Francis, Progressives Wonder how Republicans Get Elected. Daily Kos, 20 décembre. http://www.dailykos.com/story/2014/12/20/13 52183/-Duped-by-the-Media-on-Pope-Francis-Progressives-Wonder-How-Republicans-GetElected.

52. http://www.adflegal.org/about-us/who-we-are.

53. Hosanna-Tabor Evangelical Lutheran Church and School v. EEOC, 565 US (2012). Michaelson, op. cit. Pulliam Bailey S (2015) Eyeing Gay Marriage Case, Evangelicals and Catholics Become Strong Allies on Poverty. Washington Post, 14 mai. https://www.washingtonpost. com/news/acts-of-faith/wp/2015/05/14/eyeing-gay-marriage-case-evangelicals-and-catho lics-become-strong-allies-on-poverty/.

54. Pulliam Bailey, op. cit. 
55. Michaelson, op. cit. Stone A (2012) The New Religious Freedom Argument. Political Research, 30 octobre. https://www.politicalresearch.org/2012/10/30/the-new-religious-free dom-argument-gay-marriage-in-the-2012-election/. Stetzer E (2014) Religious Liberty and the Culture War. Christianity Today, 20 février. http://www.christianitytoday.com/edstet zer/2014/february/religious-liberty.html.

56. PEW (2014) Nearly Three in Ten Now See Obama Administration as Unfriendly Toward Religion, 18 septembre. http://www.pewforum.org/2014/09/22/public-sees-religions-influence-waning-2/pr_14-09-22_religionpolitics-16/.

57. Lipka M (2016) Evangelicals Increasingly Say It's Becoming Harder for Them in America. Pew Research Center, 14 juillet. http://www.pewresearch.org/fact-tank/2016/07/14/evangeli cals-increasingly-say-its-becoming-harder-for-them-in-america/.

58. Jones R, Cox D, Dionne EJ, Galston W, Cooper B et Lienesch R (2016) How Immigration and Concerns about Cultural Changes Are Shaping the 2016 Election. PPRI/Brookings, 23 juin, pp.16-17, https://www.prri.org/wp-content/uploads/2016/06/PRRI-Brookings-2016-Immigra tion-survey-report.pdf.

59. Stanton J et Shiner M (2012) New Chapter in Culture Wars. Roll Call, 9 février. http://www. rollcall.com/issues/57_93/New-Chapter-in-Culture-Wars-212260-1.html.

60. Posner S (2012) In 2012 Bishops Join Effort to Repackage Discrimination as 'Religious Freedom'. Religion Dispatches, 13 mars, http://religiondispatches.org/in-2012-bishops-joinfight-to-repackage-discrimination-as-religious-freedom/.

61. Michaelson, op. cit.

62. Lipton-Lubet S (2014) Contraceptive Coverage Under the Affordable Care Act: Dueling Narratives and Their Policy Implications. Journal of Gender, Social Policy and the Law 22(2): 360.

63. Altman A (2016) The Gospel of Ted. Time, 25 janvier, p. 34-37. Khemm S (2015) Wayward Christian Soldiers. New Republic, 1 décembre. https://www.uexpress.com/new-republic/ 2015/11/7/wayward-christian-soldiers-the-confused-religious.

64. Wolfe A (2015) How the GOP's Religious Freedom Rhetoric could undermine the Party. Politico, 8 septembre. http://www.politico.com/magazine/story/2015/09/republicans-reli gious-freedom-backfire-213130\#ixzz3vXq2imtH.

65. Brown S (2015) Lost Weekend. Church and State 68(10): 9.

66. Miller K (2013) A Guide to the Lawsuits Challenging Obamacare's Contraception Converage Requirements. Kaiser Health News, 17 septembre. http://khn.org/news/contraception-man date-challenges/.

67. http://www.usccb.org/news/2011/11-200.cfm.

68. Audi et GERSHMAN, op. cit.

69. Clarkson, Christian Right leaders escalate anti-LGBTQ threats. op. cit.

70. Lane-Steele L (2015) Masking Discrimination: How the 'Second Wave' of RFRAs can Weaken Protection for LGB Individuals. National Lawyers Guild Review 72(2): 113.

71. PFAW (2014) The Persecution Complex: The Religious Right's Deceptive Rallying Cry. San Diego Gay and Lesbian News, 16 juillet. https://sdgln.com/commentary/2014/07/16/persecu tion-complex-religious-right-deceptive-rallying-cry\#sthash.gwy0UVCS.dpbs Clarkson F (2015) When the Exception is the Rule: Christianity in the Religious Freedom Debates. The Public Eye, pp. 4-8.

72. Guo J (2015) How religious freedom laws were praised then hated then forgotten then finally resurrected. Washington Post, 3 avril. https://www.washingtonpost.com/blogs/govbeat/wp/ 
2015/04/03/how-religious-freedom-laws-were-praised-then-hated-then-forgotten-thenfinally-resurrected/.

73. Clarkson F (2015) Not all the same: Christian Right Hobby Lobbyization of state RFRAs. Political Research, 20 avril. http://www.politicalresearch.org/2015/04/20/not-all-the-samechristian-rights-hobby-lobbyization-of-state-rfras/\#sthash.uPrgKUK4.ctCXSvqi.dpuf. LANE-Steele L. op.cit. p. 114.

74. Burwell v. Hobby Lobby Stores, Inc., 134 S. Ct. 2751 (2014).

75. Nejaime D et Siegel RB (2015) Conscience Wars: Complicity-Based Conscience Claims in Religion and Politics. Yale Law Journal 124: 2516-2591.

76. Zubik v. Burwell, 136 S. Ct. 1557 (2016) et les sept autres affaires pendantes, plus Catholic Healthcare System v. Burwell et Michigan Catholic Conference v. Burwell.

77. Elane Photography, LLC v. Willock, 309 P.3d 53 (NM 2013).

78. State of Washington v. Arlene's Flowers, 187 Wn.2d 804 (2017). Craig v. Masterpiece Cakeshop, Inc, 2015 COA 115.

79. Jack v. Azucar Bakery, Colorado Civil Rights Commission Determination, Charge No. P20140069x (2015).

80. La Cour devait répondre de la plainte de quatorze citoyens contre les lois de plusieurs États américains sur le mariage qui réaffirmaient son caractère hétérosexuel. Les plaignants considéraient que ces lois constituaient une violation du $14^{\mathrm{e}}$ amendement fédéral. Et la Cour suprême leur a donné raison par cinq voix contre quatre.

81. Le texte en discussion intitulé Oklahoma Religious Freedom Restoration Act de 2015 (OK SB 440 2016) élargit la définition de l'organisation religieuse, exemptée au nom de ses croyances concernant « le sexe, le genre ou la sexualité », d'admettre la validité légale d'un mariage homosexuel ou de procurer ses services ou ses offres d'emplois à des couples homosexuels. L'organisation religieuse redéfinie, inclut les entreprises privées « opérant de manière cohérente avec des convictions religieuses sincères ». De nouveaux amendements au projet ont été introduits en 2016: précision de ce que l'intérêt vital de l'Etat ne peut jamais permettre de forcer quelqu'un à participer à une cérémonie de mariage (homosexuel) ou à fournir ses services en vue de ce genre d'activités (OK HB1371 2016); précision de ce que l'exercice de la religion inclut dans sa définition la faculté d'agir ou de refuser d'agir en accord avec sa foi (OK SB 898 2016).

82. The First Amendment Defense Act (H.R. 2802, S. 1598).

83. http://www.usccb.org/news/2015/15-096.cfm.

84. Sur toute l'activité concernant la lutte des évêques contre la légalisation du mariage homosexuel, cf sur le site de la conférence des évêques, la page consacrée à cette question http:// www.usccb.org/issues-and-action/marriage-and-family/marriage/promotion-and-defense-ofmarriage/defense-of-marriage-in-the-news.cfm.

85. https://www.ncronline.org/news/politics/trumps-pledge-catholics-ill-be-there-you

86. Trump D (2016) Campaign Speech at Dordt College. The 405 Media, 23 janvier. http:// the405media.com/2016/01/23/donald-trump-sioux-center-ia-123/.

87. Goldberg M (2017) Donald Trump, the Religious Right's Trojan Horse. New York Times, 27 janvier. https://www.nytimes.com/2017/01/27/opinion/sunday/donald-trump-the-religiousrights-trojan-horse.html?_r=0. 
88. Goodstein L et Shear M (2017) and Mark Shear, “Trump's Order on Religious Liberty Pleases a Few, but Lets Down Many Conservatives," New York Times, 4 mai. https://www.nytimes. com/2017/05/04/us/politics/religious-organizations-executive-order-trump.html?_r=0.

89. Presidential Executive Order Promoting Free Speech and Religious Liberty, 4 mai 2017, https://www.whitehouse.gov/presidential-actions/presidential-executive-order-promotingfree-speech-religious-liberty/.

90. Shellnutt K (2017) Trump's Religious Liberty Order Doesn't Answer Most Evangelicals' Prayers. Christianity Today, 4 mai.

http://www.christianitytoday.com/news/2017/may/trump-religious-liberty-order-johnsonamendment-ndop-prayer.html.

91. Ibid.

92. Ibid.

93. USCCB (2017) USCCB Chairmen Ask President Trump to Fulfill His Promise to Protect Religious Liberty. 16 février. http://www.usccb.org/news/2017/17-040.cfm.

94. Posner S (2017). The Christian Legal Army Behind 'Masterpiece Cakeshop'. The Nation, 28 novembre,

https://www.thenation.com/article/the-christian-legal-army-behind-masterpiece-cakeshop/

95. Pulliam Bailey S (2018) HHS is Targeting Health Workers' Religious Objections. Here's Why. Washington Post, 19 janvier. https:/www.washingtonpost.com/news/acts-of-faith/wp/ 2018/01/18/a-short-history-of-why-hhs-cares-about-doctors-religious-objections/?utm_ term $=.2 \mathrm{~b} 08 \mathrm{e} 0 \mathrm{ba} 41 \mathrm{~b} 6$.

96. Ibid. Goodstein L (2017) Some Worry about Judicial Nominee's Ties to a Religious Group. New York Times, 28 septembre, https://www.nytimes.com/2017/09/28/us/amy-coney-barrettnominee-religion.html?_r=0

97. President Donald J. Trump Announces Eighth Wave of Judicial Candidates, 28 septembre 2017, https://www.whitehouse.gov/presidential-actions/president-donald-j-trump-announceseighth-wave-judicial-candidates/.

98. Une étude du Pew Research Center réalisée en 2012 en pleine controverse sur le mandat contraceptif révélait que seuls $8 \%$ des catholique interrogés la jugeaient immorale.

99. Gayte M (2016) La liberté religieuse contre les femmes et les homosexuels : nouveau chapitre des guerres culturelles aux Etats-Unis ? Estudos de Religião 30(1) : 75-91.

rs: Complicity-Based Conscience Claims in Religion and Politics. Yale Law Journal 124: 2516-2591.

100. Remarks by President Trump at the Faith and Freedom Coalition's Road to Majority Conference. 8 June 2017. https://www.whitehouse.gov/the-press-office/2017/06/08/remarkspresident-trump-faith-and-freedom-coalitions-road-majority.

\section{Références}

Byrnes T (1991) Catholic Bishops in American Politics. Princeton, NJ: Princeton University Press. D'Antonio WV (2012) Catholic Bishops and the Electoral Process in American Politics. In: CAFARDI N (ed) (2012) Voting and Holiness: Catholic Perspectives On Political Participation. New York: Paulist Press.

Diamond S (2000) Not by Politics Alone. The Enduring Influence of the Christian Right. New York: Guilford Press.

Enda J (2012) US Bishops' Roman Connection. Conscience 33 (3): 14-18. 
Gayte M (2016) La liberté religieuse contre les femmes et les homosexuels : nouveau chapitre des guerres culturelles aux Etats-Unis ? Estudos de Religião 30(1): 75-91

Gayte M, Chelini-Pont B et Rozell M (eds) (2018) Catholics and US Politics After the 2016 Elections Understanding the 'Swing Vote'. New York: Palgrave Macmillan.

Heyer K, Rozell M et Genovese M (eds) (2008) Catholics and Politics. The Dynamic Tension between Faith and Power. Washington DC: Georgetown University.

Hunter JD (1991) Culture Wars: The Struggle to Define America. New York: Basic Books.

Klemp N (2012) The Morality of Spin. Virtue and Vice in Political Rhetoric and the Christian Right. Lanham, MD: Rowman and Littlefield.

Lane-Steele L (2015) Masking discrimination: How the "second wave" of RFRAs can weaken protection for LGB individuals. National Lawyers Guild Review 72(2).

Lipton-Lubet S (2014) Contraceptive Coverage Under the Affordable Care Act: Dueling Narratives and Their Policy Implications. Journal of Gender, Social Policy and the Law 22(2).

Marley DJ (2006) Riding in the Back of the Bus. The Christian Right's Adoption of Civil Rights Movement Rhetoric. In: Romano R et Raiford L (eds) The Civil Rights Movement in American Memory. Athens: The University of Georgia Press.

Marlin GJ (2004) The American Catholic Voter. 200 Years of Political Impact. South Bend: St Augustine's Press.

McCarthy J (1997) The Globalization of Social Movement Theory. In: Smith J, Chatfield C et Ron Pagnucco R (eds) Transnational Social Movements and Global Politics: Solidarity beyond the State. Syracuse, NY: Syracuse University Press.

Miceli M (2005) Morality Politics vs. Identity Politics: Framing Processes and Competitions among Christian Right and Gay Social Movement Organizations. Sociological Forum 20(4).

Michaelson J (2016) Redefining Religious Liberty: The Covert Campaign against Civil Rights. Political Research, 20 mars. https://www.politicalresearch.org/2013/03/21/redefining-reli gious-liberty-the-covert-campaign-against-civil-rights/

Miller P (2014) Good Catholic: The Battle over Abortion in the Catholic Church. Berkeley: University of California Press.

Nejaime D et Siegel RB (2015) Conscience Wars: Complicity-Based Conscience Claims in Religion and Politics. Yale Law Journal 124: 2516-2591

Ross Sammon M (2008) The Politics of the US Catholic Bishops: The Centrality of Abortion.

In Heyer K, Rozell M et Genovese M (eds) Catholics and Politics. The Dynamic Tension between Faith and Power. Washington DC: Georgetown University Press, pp. 11-26.

Sullivan A (2014) The Party Faithful: How and Why Democrats Are Closing the God Gap. New York: Scribner.

Swole Cahill L (2012) Voting and Living the Common Good. In: Cafardi, op. cit.

Wilcox C et Robinson C (1996) Onward Christian Soldiers? The Religious Right in American Politics. Boulder, CO: Westview Press.

Wilcox C, Merolla LM et Beer D (2006) Saving Marriage by Banning Marriage: The Christian Right Finds a New Issue in 2004. In: Green J, Rozell M et Wilcox C (eds) The Values Campaign?: The Christian Right and the 2004 Elections. Washington DC: Georgetown University Press.

Young N (2015) We Gather Together: The Religious Right and the Problem of Interfaith Politics. New York: Oxford University Press. 